\title{
Physical activity and mortality among Norwegian women - the Norwegian Women and Cancer Study
}

This article was published in the following Dove Press journal:

Clinical Epidemiology

28 July $201 \mathrm{I}$

Number of times this article has been viewed

\author{
Kristin Benjaminsen Borch' \\ Tonje Braaten' \\ Eiliv Lund' \\ Elisabete Weiderpass $1,2,3,4$ \\ 'Department of Community \\ Medicine/University of Troms $\varnothing$. \\ Tromsø, Norway; ${ }^{2}$ Department \\ of Medical Epidemiology and \\ Biostatistics, Karolinska Institutet, \\ Stockholm, Sweden; ${ }^{3}$ Cancer Registry \\ of Norway, Oslo, Norway; ${ }^{4}$ Folkhälsan \\ Research Centre, Samfundet \\ Folkhälsan, Helsinki, Finland
}

Correspondence: Kristin Benjaminsen Borch

Department of Community Medicine/ University of Tromsø,

9037 Tromsø, Norway

$\mathrm{Tel}+4777645443$

Fax +47 776 4483

Email kristin.benjaminsen.borch@uit.no
Background: Physical activity (PA) and its relationship with all-cause mortality suggest a strong and consistent inverse association. This study prospectively investigated the association between PA level and mortality among participants of the Norwegian Women and Cancer (NOWAC) Study.

Methods: A total of 66,136 NOWAC participants were followed-up until December 31st 2008. PA level and possible confounding factors were obtained through a self-administered questionnaire at enrolment. Cox proportional hazards regression was used to calculate adjusted relative risks (RRs) and 95\% confidence intervals (CIs) for all-cause, cardiovascular disease (CVD) and cancer mortality and PA levels defined from 1 to 10 on a global scale.

Results: PA levels 1-4 were associated with a significantly increased risk of all-cause mortality (level $1 \mathrm{RR}=2.35$; 95\% CI: 1.94-2.84, level $2 \mathrm{RR}=1.71 ; 95 \% \mathrm{CI}$ : $1.45-2.00$, level 3 $\mathrm{RR}=1.30 ; 95 \% \mathrm{CI}: 1.14-1.49$, level $4 \mathrm{RR}=1.07$; 95\% CI: 0.95-1.22), compared with PA level 5. CVD mortality risk increased in PA levels $1-3$ (level $1 \mathrm{RR}=3.50$; 95\% CI: 2.41-5.10, level $2 \mathrm{RR}=1.50 ; 95 \% \mathrm{CI}: 0.99-2.25$, level $3 \mathrm{RR}=1.12 ; 95 \% \mathrm{CI}: 0.79-1.60$ ) as did cancer mortality risk $(\mathrm{RR}=1.32 ; 95 \% \mathrm{CI}: 0.96-1.81, \mathrm{RR}=1.48 ; 95 \% \mathrm{CI}: 1.19-1.84, \mathrm{RR}=1.26 ; 95 \%$ CI: $1.06-1.50$, respectively). The magnitude of the associations was consistent across strata of age, smoking, and body mass index. The population attributable fractions for PA levels 1-4 were: all-cause mortality, $11.5 \%$; CVD mortality, $11.3 \%$; cancer mortality, $7.8 \%$.

Conclusion: There is a significant trend of increased risk of all-cause, CVD and cancer mortality in relation to low PA levels among Norwegian women.

Keywords: physical activity, mortality, cardiovascular disease, cancer, Norway, women

\section{Introduction}

Physical activity (PA) and its relationship with mortality have been investigated in several studies $^{1-9}$ and a strong and consistent inverse association between PA and all-cause mortality has been suggested. National ${ }^{10}$ and international ${ }^{11}$ public health recommendations for PA are based on evidence linking a physically active lifestyle to reduced mortality. One review on PA and all-cause mortality concluded that there is an inverse dose-response relationship between total amount of PA and all-cause mortality ${ }^{12}$ and another review suggested that physically active men and women have a 34\% lower premature mortality risk. ${ }^{2}$ In a study of Norwegian and Swedish women below the age of 60 years, PA at baseline substantially reduced all-cause mortality. ${ }^{13}$ It has not been convincingly documented whether this is also applicable to specific causes of death such as cardiovascular disease (CVD) and cancer, and the degree to which PA independently contributes to mortality risk has not been explored thoroughly. 
The aim of the present study was therefore to investigate what impact PA level has on all-cause mortality in middleaged women, to specify the relative risk (RR) of death due to CVD and cancer, and to calculate the population attributable fraction (PAF) of all-cause, CVD and cancer mortality among women in Norway.

\section{Methods}

\section{Study population}

The Norwegian Women and Cancer (NOWAC) Study is a population-based prospective cohort study which enrolled the first participants in 1991. A more detailed description of the cohort and design of the NOWAC Study has previously been described, ${ }^{14}$ as has external validity. ${ }^{15}$ Briefly, from 1991-1997 a random sample of women aged 30-70 years was drawn from the National Population Register in Norway and invited to participate in the study. All women received a letter of invitation requesting informed consent and a comprehensive eight-page self-administered questionnaire. Of those invited, 102,540 women (57\%) completed and returned the questionnaire. ${ }^{14}$ Due to study logistics, the first enrolment was divided into 24 mailings over 7 years. For the present analysis, we collected baseline information from the women who answered the first mailing in 1996-1997 (37,899 women), and those who answered a second mailing in 1998 (46,965 women), for a total of 84,864 women. We excluded 53 women with a reported date of emigration or death that was before the date of recruitment. We excluded 8137 women with missing information on PA level at cohort enrolment and another 10,538 women due to lack of information on other covariates examined. Hence 66,136 women were eligible for inclusion in our present analysis. The Regional Ethical Committee and the Norwegian Data Inspectorate approved the study.

\section{Assessment of PA level}

PA level at age 14 years, 30 years and at the time of cohort enrolment were assessed by self-report on a scale of 1 to 10 . PA was defined in the questionnaire as follows: "By physical activity we mean activity both at work and outside work, at home, as well as training/exercise and other physical activity, such as walking, etc. Please mark the number that best describes your level of physical activity; 1 being very low and 10 being very high". The PA scale used for this study refers to the total amount of PA, including different domains, frequencies, durations and intensities in one global score, and have been recently validated. A moderate but significant $(P<0.001)$ Spearman's rank correlation coefficient was found, in the range of 0.36 to 0.46 , between the PA scale and outcomes from objective measures of a combined sensor monitoring heart rate and movement, and appeared valid to rank PA in a Norwegian population of women.

\section{Covariates}

Information collected at cohort enrolment included age; duration of education; height; weight; smoking history (including smoking status: current, former, never; duration and quantity of cigarettes smoked/day); alcohol consumption (grams/day); detailed information on dietary habits and intake of certain food items which was used to calculate total energy intake (Kcal/day); parity; use of oral contraceptives or postmenopausal hormone therapy (HT); age at menarche; and age at menopause. ${ }^{16-18}$ History of heart failure, myocardial infarction, angina pectoris, diabetes mellitus, fibromyalgia, rheumatism and hypertension were self-reported. Information on cancer diagnosis before cohort enrolment was obtained through linkages to the database of the Cancer Registry of Norway.

\section{Follow-up}

Person-years were calculated from the start of follow-up until the date of emigration, death, or end of the study period (December 31st 2008), whichever occurred first. We obtained information on date of emigration and death from the National Population Register. Cause of death was obtained from the National Register for Causes of Death, and categorized into specific causes of death: CVD deaths (ICD-10: I00-I99), including stroke, coronary heart disease, and other vascular causes; cancer deaths (ICD-10: C00-D48) and other-cause deaths (from causes other than CVD and cancer). The combination of the aforementioned categories was classified as all-cause mortality.

\section{Statistical analysis}

Characteristics of the study population are presented as means and standard deviations, and frequency tabulations over the different PA levels. Cox proportional hazards regression, with follow-up time as the time scale, was used to calculate adjusted hazard ratios (HRs) (interpreted as estimates of RRs) and corresponding 95\% confidence intervals (CIs), which were used to examine the association between PA level and all-cause, CVD and cancer mortality. The proportional hazard assumption was checked using Schoenfeld residuals and Kaplan-Meier plots, which suggested no evidence of deviation from proportionality. The initial survival analyses were done by PA level adjusted for age and further built up in multivariate models, with covariates added in a backward 
stepwise manner to take possible confounders into account. Additional analyses were conducted separately by age group, body mass index (BMI) and history of CVD, diabetes mellitus or prevalent cancer to determine whether associations were stronger in these subgroups. The analyses were repeated excluding women with CVD, diabetes mellitus or prevalent cancer to minimize the potential effect of reverse causality. PA level at baseline was entered into the Cox regression models as a categorical variable with 10 categories, in order to reflect the study scale. PA level 5 (moderate PA level) in the study scale was used as the reference category, compared to PA levels 1-4 (low PA levels), and 6-10 (high PA levels).

Covariates examined in the multivariate Cox regression models were age (40-49, 50-59, $\geq 60$ years); BMI (weight in kilograms divided by height in centimeters squared, $<18.5$, $18.5-24,25-29, \geq 30$ ) with the second category as the reference; height $(\mathrm{cm})$; total energy intake (Kcal/day). Smoking status (never, former, current), smoking duration (years) and quantity smoked (pack-years $=$ the total number of years a smoker smoked 20 cigarettes/day) were combined in the analysis as one variable (age at start $\geq 20$ years and current smoker, age at start $\geq 20$ years and former smoker, age at start $<20$ years, $0-19$ pack-years and current smoker, age at start $<20$ years and former smoker, and finally $\geq 20$ packyears, age at start $<20$ years and current smoker). Duration of education (0-9, 10-12 and $\geq 13$ years) and menopausal status at enrolment (postmenopausal yes/no) were further included. Baseline information on menopausal status was available for 50,875 women, and the remaining women were either perimenopausal $(\mathrm{N}=6730)$, hysterectomized at $<53$ years of age $(\mathrm{N}=2150)$, HT users $<53$ years of age $(\mathrm{N}=5477)$ or had missing information $(\mathrm{N}=1828)$. Use of HT was registered as ever or never, and self-reported disease (yes/no to any of the following: heart failure, myocardial infarction, angina pectoris, diabetes mellitus, fibromyalgia, rheumatism or hypertension) was also included in order to analyze each disease as a separate covariate. Alcohol intake was divided into four categories (none, low $=0.1-3.9$ grams $/$ day, medium $=4.0-10.0$ grams $/$ day and high intake $>10.0$ grams/day) with low intake as reference category, and information on age at first birth $(<20$, $20-25,>25$ years $)$ and parity $(0,1,2, \geq 3$ children) was combined into one variable.

PAF was interpreted as the proportional reduction in the average population mortality risk that would occur if low PA levels were eliminated from the population, assuming that the distribution of the adjustment variables remained unchanged. ${ }^{19}$ PAF was estimated using the following equation: $\mathrm{PAF}=\left(\mathrm{P}_{\mathrm{e}}(\mathrm{RR}-1) / 1+\mathrm{P}_{\mathrm{e}}(\mathrm{RR}-1)\right)$, where $\mathrm{P}_{\mathrm{e}}$ is the proportion of the exposed population, and $\mathrm{RR}$ is the risk estimate for the exposed compared to the unexposed population in the final multivariate proportional hazards regression model, including all aforementioned covariates. The cut-off point a priori for the exposed population was set at PA level 4 or less, which divided the study population in two: exposed (PA levels 1-4) and unexposed (PA levels 5-10). Analyses were conducted using STATA version 11.0, special edition (StataCorp, Lakeway Drive, College Station, TX).

\section{Results}

Characteristics of the study population at cohort enrolment and mortality during follow-up are given in Table 1 . During 705,980 person-years of follow-up from 1996 to December 31st 2008, 2572 deaths were reported. The mean age of the participants at baseline was 50.5 years (standard deviation, $41-70$ years) (Table 1).

Self-reported PA level and health-related characteristics of study women are shown in Table 2 by PA level. Almost $30 \%$ of the women reported low PA levels (levels $1-4$ ), and women who reported moderate to high PA levels (levels 5-10) tended to be leaner and had a higher total energy intake. Women with a PA level of 1 were more often current smokers compared to women with moderate to high PA levels. CVD was reported by $13 \%$ of study women, and this proportion was lower among women reporting moderate to high PA levels compared with those who reported low PA levels. A similar pattern was observed for diabetes mellitus, which was reported by $1.5 \%$ of study women. A total of $4.2 \%$ of women were diagnosed with cancer before cohort enrolment, and there was a higher percentage of prevalent cancer among women with a PA level of 1 (Table 2).

Survival analysis of all-cause mortality with adjustments for different covariates showed a significantly increased

Table I Characteristics of the study population at baseline and mortality during follow-up - The Norwegian Women and Cancer Study

\begin{tabular}{ll}
\hline Characteristics & \\
Number of women & 66,136 \\
Mean age at entry (SD), years & $50.5(6.55)$ \\
Mean person-years of follow-up (SD) & $10.70(1.48)$ \\
Total person-years at risk & 705,980 \\
Number of deaths in the follow-up period & 2572 \\
Number of deaths by cause & \\
CVD & 401 \\
Cancer & 1584 \\
Other causes & 587 \\
\hline
\end{tabular}

Abbreviations: SD, standard deviation; CVD, cardiovascular disease. 
Table 2 Physical activity (PA) level and health-related characteristics according to PA level at cohort enrolment in 1996-1998 among 66, 136 women from the Norwegian Women and Cancer Study

\begin{tabular}{|c|c|c|c|c|c|c|c|c|c|c|}
\hline \multirow[t]{2}{*}{ Characteristics } & \multicolumn{10}{|c|}{ PA level } \\
\hline & 1 & 2 & 3 & 4 & 5 & 6 & 7 & 8 & 9 & 10 \\
\hline $\mathrm{N}$ & 949 & 2421 & 5810 & 9713 & 16,862 & $|2,02|$ & 9828 & 5489 & 1896 & 1147 \\
\hline PA level, \% & 1.43 & 3.7 & 8.8 & 14.7 & 25.5 & 18.2 & 14.9 & 8.3 & 2.9 & 1.73 \\
\hline Age, mean (years) & 53.6 & 52 & 51.3 & 50.9 & 50.5 & 50 & 49.8 & 50 & 50.5 & 51.4 \\
\hline Duration of education, mean (years) & 10.4 & 11.3 & 11.8 & 12 & 11.9 & 12 & 12.3 & 11.9 & 11.5 & 10.8 \\
\hline BMI, mean $\left(\mathrm{kg} / \mathrm{m}^{2}\right)$ & 26.6 & 26.5 & 25.8 & 25.3 & 24.6 & 24.1 & 23.7 & 23.5 & 23.4 & 23.5 \\
\hline Height, mean $(\mathrm{cm})$ & 165.1 & 165.9 & 165.9 & 166 & 166.2 & 166.3 & 166.4 & 166.1 & 166.2 & 165.6 \\
\hline Weight, mean $(\mathrm{kg})$ & 72.7 & 72.9 & 71.1 & 69.7 & 68 & 66.7 & 65.7 & 65 & 64.7 & 64.6 \\
\hline Current smoker (\%) & 44.9 & 39.4 & 34.5 & 32.5 & 32.3 & 30 & 28.6 & 30.9 & 32.3 & 36.7 \\
\hline Alcohol consumption (grams/day) & 3.0 & 2.9 & 3.4 & 3.3 & 3.2 & 3.3 & 3.4 & 3.2 & 3.0 & 2.6 \\
\hline Total energy intake, mean (Kcal/day) & 1472 & 1515 & 1548 & 1592 & 1626 & $166 \mid$ & 1686 & 1713 & 1738 & 1758 \\
\hline Nulliparous (\%) & 9.5 & 10.0 & 9.5 & 9.0 & 8.1 & 7.7 & 7.8 & 9.1 & 7.5 & 8.4 \\
\hline First birth, mean age (years) ${ }^{\mathrm{a}}$ & 23.5 & 24 & 23.9 & 23.9 & 24 & 24 & 23.8 & 23.5 & 23.3 & 23.9 \\
\hline Hormone therapy use at enrolment (\%) & 36.8 & 35.6 & 33.8 & 32.5 & 32.1 & 30.1 & 29.3 & 27.9 & 29.6 & 26.7 \\
\hline Postmenopausal at enrolment (\%) ${ }^{\mathrm{b}}$ & 74.7 & 65.2 & 58.8 & 56.5 & 54.0 & 49.5 & 48.4 & 50.5 & 53.0 & 57.8 \\
\hline Cardiovascular disease $(\%)^{c}$ & 27.8 & 23.3 & 18.7 & 16.4 & 13.2 & 11.3 & 10.4 & 10.3 & 9.3 & 11.2 \\
\hline Diabetes mellitus (\%) & 4.6 & 3.7 & 2.3 & 1.5 & 1.5 & 1.3 & 0.9 & 1.0 & 1.2 & 2.3 \\
\hline Cancer (\%) ${ }^{\mathrm{d}}$ & 7.1 & 6.0 & 5.2 & 4.4 & 4.4 & 3.7 & 3.6 & 3.4 & 3.6 & 4.5 \\
\hline
\end{tabular}

Notes: aAmong those who had children; 'Postmenopausal status based on 50,875 women, excluded from this category were 629 I women classified as perimenopausal, 2 I 50 with hysterectomy $<53$ years of age and 5477 hormone therapy users $<53$ years of age; 'Self-reported cardiovascular diseases defined as heart failure, myocardial infarction, angina pectoris, and hypertension; Information on cancer diagnosis before cohort enrolment obtained from the Cancer Registry of Norway.

risk of mortality for women with low PA levels (level 1 $\mathrm{RR}=2.35 ; 95 \% \mathrm{CI}: 1.94-2.84$, level $2 \mathrm{RR}=1.71 ; 95 \% \mathrm{CI}$ : 1.45-2.00, level $3 \mathrm{RR}=1.30 ; 95 \% \mathrm{CI}: 1.14-1.49$, level 4 $\mathrm{RR}=1.07 ; 95 \% \mathrm{CI}: 0.95-1.22$ ), compared with women with a moderate PA level (Table 3). The association between PA level and all-cause mortality showed a significant overall trend $(P<0.001)$ of reduced mortality risk in women with moderate to high PA levels compared to women with low PA levels. However, the shape of the association seemed nonlinear; more like an L-shape, indicating no additive effect on mortality beyond the moderate PA level (Figure 1).

CVD mortality risk for women with a PA level of 1 was found to be 3.5 times higher (95\% CI: 2.41-5.10) than for women with a moderate PA level (Table 3 ). PA levels of 2 , 3 and 4 separately showed an increased risk as well (though not significant), albeit not as high as for PA level 1 (Table 3 ). The overall trend for CVD mortality risk in women with low PA levels was significant $(P<0.001)$. The results indicated a significant trend $(P=0.002)$ of increased risk of cancer mortality for women with decreasing PA levels. Separate results for PA levels 1, 2 and 3 were $\mathrm{RR}=1.32(95 \% \mathrm{CI}$ : 0.96-1.81), 1.48 (95\% CI: 1.19-1.84) and 1.26 (95\% CI: $1.06-1.50)$, respectively.

Participants with self-reported diseases or prevalent cancer at baseline were included and adjusted for in the main analytical model. The results of sensitivity analyses after exclusion of all women with CVD, diabetes mellitus, or prevalent cancer, did not significantly change the results (data not shown) and these participants were therefore retained in the final analysis. Survival analysis was conducted in strata of age, BMI and history of CVD, diabetes mellitus and cancer. No evidence of a differential effect of PA level on mortality according to age, BMI, or history of included disease was found, indicating that the increased mortality risk found in the main survival analyses are independent of these factors (data not shown).

The proportion of the population with low PA levels was $29 \%$ with an adjusted RR of 1.45 (95\% CI: $1.34-1.57)$ for all-cause mortality, 1.44 (95\% CI: 1.17-1.76) for CVD mortality and 1.29 (95\% CI: 1.16-1.43) for cancer mortality for the exposed compared to the unexposed population. Our results showed that $11.5 \%$ of all-cause mortality, $11.3 \%$ of CVD mortality and $7.8 \%$ of cancer mortality was attributable to low PA levels.

\section{Discussion}

We found a significant trend of reduced all-cause mortality with increasing PA level, although the shape of the inverse association was non-linear and more L-shaped, with no additional effect beyond the moderate PA level of 5 . Women reporting low PA levels (ie, PA levels 1-4) had a significantly increased risk of all-cause mortality, with a stronger association for CVD than for cancer-related mortality. Separate subgroup analyses indicated that the findings were 
Table 3 Relative risks (RRs) and 95\% confidence intervals (Cls) of the association between physical activity (PA) level and allcause, cardiovascular disease and cancer mortality among 66, I 36 women from the Norwegian Women and Cancer Study

\begin{tabular}{|c|c|c|c|}
\hline \multirow[t]{2}{*}{ PA level ${ }^{\mathrm{a}}$} & \multirow{2}{*}{$\begin{array}{l}\mathbf{N} \text { of } \\
\text { deaths }\end{array}$} & \multirow{2}{*}{$\begin{array}{l}\text { Age-adjusted } \\
\text { RR }(95 \% \mathrm{CI})\end{array}$} & \multirow{2}{*}{$\begin{array}{l}\text { Multivariate } \\
\text { RR }(95 \% \mathrm{Cl})\end{array}$} \\
\hline & & & \\
\hline \multicolumn{4}{|c|}{ All-cause mortality } \\
\hline I & 139 & $3.17(2.64-3.82)$ & $2.35(1.94-2.84)$ \\
\hline 2 & 203 & $2.04(1.74-2.39)$ & I.7I (1.45-2.00) \\
\hline 3 & 321 & $1.40(1.22-1.60)$ & $\mathrm{I} .30(\mathrm{I} .14-\mathrm{I} .49)$ \\
\hline 4 & 402 & $1.09(0.96-1.23)$ & $1.07(0.95-1.22)$ \\
\hline 5 & 621 & 1.0 & 1.0 \\
\hline 6 & 342 & $0.80(0.70-0.93)$ & $0.83(0.73-0.95)$ \\
\hline 7 & 276 & $0.81(0.70-0.93)$ & $0.86(0.75-0.99)$ \\
\hline 8 & 170 & $0.87(0.74-1.03)$ & $0.89(0.75-1.06)$ \\
\hline 9 & 54 & $0.76(0.58-1.00)$ & $0.76(0.58-1.01)$ \\
\hline 10 & 44 & $0.94(0.69-1.28)$ & $0.86(0.63-1.17)$ \\
\hline$P$ for trend & & $<0.001$ & $<0.001$ \\
\hline \multicolumn{4}{|c|}{ Cardiovascular disease mortality } \\
\hline I & 44 & $6.04(4.21-8.66)$ & $3.50(2.4 I-5.10)$ \\
\hline 2 & 32 & $2.10(1.37-3.08)$ & $1.50(0.99-2.25)$ \\
\hline 3 & 46 & $1.31(0.92-1.86)$ & I.I2 (0.79-I.60) \\
\hline 4 & 60 & $1.10(0.78-1.49)$ & $1.00(0.72-1.39)$ \\
\hline 5 & 92 & 1.0 & 1.0 \\
\hline 6 & 48 & $0.77(0.5-1.09)$ & $0.83(0.58-I .17)$ \\
\hline 7 & 40 & $0.80(0.55-1.17)$ & $0.89(0.61-1.30)$ \\
\hline 8 & 29 & I.0I (0.67-I.55) & $\mathrm{I} .07(0.7 \mathrm{I}-\mathrm{I} .63)$ \\
\hline 9 & 5 & $0.47(0.19-1.15)$ & $0.47(0.19-1.15)$ \\
\hline 10 & 5 & $0.70(0.28-I .7 I)$ & $0.62(0.25-1.53)$ \\
\hline$P$ for trend & & $<0.001$ & $<0.001$ \\
\hline \multicolumn{4}{|c|}{ Cancer mortality } \\
\hline I & 43 & $1.60(1.17-2.19)$ & $1.32(0.96-1.81)$ \\
\hline 2 & 105 & I.67 (I.35-2.08) & I.48 (I.19-I.84) \\
\hline 3 & 192 & $1.32(1.11-1.57)$ & $1.26(1.06-1.50)$ \\
\hline 4 & 252 & $\mathrm{I} .07(0.9 \mathrm{I}-\mathrm{I} .25)$ & $\mathrm{I} .07(0.9 \mathrm{I}-\mathrm{I} .25)$ \\
\hline 5 & 399 & 1.0 & 1.0 \\
\hline 6 & 234 & $0.85(0.73-1.00)$ & $0.88(0.75-1.03)$ \\
\hline 7 & 188 & $0.85(0.72-1.01)$ & $0.90(0.76-1.07)$ \\
\hline 8 & 111 & $0.88(0.72-1.09)$ & $0.92(0.74-1.13)$ \\
\hline 9 & 37 & $0.82(0.58-I .14)$ & $0.84(0.60-1.17)$ \\
\hline 10 & 23 & $0.78(0.5 \mathrm{I}-\mathrm{I} .18)$ & $0.75(0.49-1.15)$ \\
\hline$P$ for trend & & $<0.001$ & $<0.002$ \\
\hline
\end{tabular}

Notes: Data are adjusted in the multivariate model for age at cohort enrolment, body mass index, height, smoking status, years of smoking, amount of smoking, alcohol intake, menopausal status, age at first birth, parity, hormone therapy use, cardiovascular disease diabetes mellitus and prevalent cancer. Reference level for body mass index is normal weight and for alcohol intake the reference category is low intake. Duration of education in years and total energy intake was tested in the model, but not included

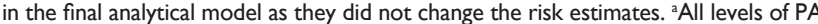
from the study scale, I being the lowest PA level, and 10 being the highest.

independent of age, BMI and history of CVD, diabetes mellitus, or cancer. PAF calculation showed that $11.5 \%$ of all-cause deaths, $11.3 \%$ of CVD deaths and $7.8 \%$ of cancer deaths were attributable to low PA levels.

Strengths of our study include the prospective design, the large sample size, relatively high response rates, almost complete follow-up (99.9\%) with almost 11 years of mean

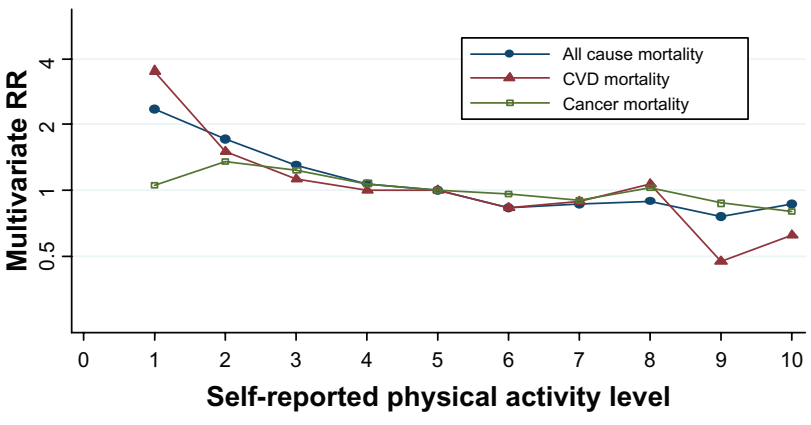

Figure I Multivariate relative risks (RRs) of all cause, CVD and cancer mortality and physical activity (PA) level.

Abbreviation: CVD, cardiovascular disease.

follow-up time, and the evaluation of cause-specific mortality. The study information on HT use ${ }^{20}$ and dietary habits ${ }^{21,22}$ are considered valid. The NOWAC cohort is representative of the Norwegian female population in the studied age groups, and there was no major selection bias that would invalidate a PAF calculation. ${ }^{15}$

Despite the different methods that exist to measure PA level, a substantial amount of evidence from observational studies confirms an inverse relationship between low PA levels and increased mortality risk in women., ${ }^{2,12,23,24}$ Our findings are in line with these observations for all-cause, CVD and cancer mortality. Our observations suggested that the relationship is non-linear, with the greatest benefit to be had by changing from a PA level of 1 to any level between 2 and 5 , which has also been confirmed in other studies. ${ }^{23}$

Physical inactivity is considered the fourth leading cause of death worldwide. ${ }^{25}$ In our study, assuming causality, the PAF for low PA levels among women was $11.5 \%$ for all-cause, $11.3 \%$ for CVD and $7.8 \%$ for cancer mortality, which is substantial. For modifiable factors like PA level, PAF is useful for the planning of public health strategies and interventions. The Nurses' Health study in the United States found a PAF of $17 \%$ for all-cause, $28 \%$ for CVD and $9 \%$ for cancer mortality among women reporting a duration of PA of less than 30 minutes per day. ${ }^{26}$ In a global health repor $\mathrm{t}^{25}$ the estimated PAF of mortality was $8 \%$ for physical inactivity in high-income countries. However, underestimation is likely as PA level measures were limited. ${ }^{25}$ Our results indicated a significant, but weaker trend in the association between PA and cancer mortality than CVD mortality. Cancer develops over several years before symptoms appear and are diagnosed. This suggests that duration of follow-up is critical and must be of a certain length to capture the magnitude of an association. Among environmental risk factors for cancer incidence, lifestyle factors such as tobacco use, poor nutrition, obesity and physical inactivity, which we adjusted 
for in our study, account for about one-third of all cancers. ${ }^{27}$ We further adjusted for hormone use (oral contraceptives and HT) and reproductive factors. Furthermore, our findings are in line with several other studies that suggested an association between reduced cancer mortality and PA level. ${ }^{28-30}$

Our study has some limitations. PA level in our study was self-reported and assessed at baseline only. Changes in PA level over time could introduce bias. Therefore the interpretation of our results should take into consideration the possibility of changes in behaviour over time: if anything PA levels decrease with age, ${ }^{31}$ and therefore our results are probably underestimates of the true PAF. Measurement errors are inevitable and self-report is a crude measure of PA level, which necessarily entails some degree of misclassification. ${ }^{32}$ A known problem of self-reporting of a desirable behaviour like PA is the tendency to report levels that are higher than one really has. ${ }^{33}$ However, the size of the NOWAC cohort limits the use of other more accurate measures such as accelerometer or heart rate monitors. The results from our validation study indicated that the scale of 10 PA levels is sufficient to differentiate between levels of the total amount of PA. Although the challenge of equating different total amounts of PA to the different PA levels on the scale remains, the 10 categories represent ordered levels of the total amount of PA which have a strong predictive value in relation to mortality. Studies have shown that a higher total volume of PA is clearly associated with decreased all-cause mortality. ${ }^{12,34}$ Total volume of PA refers to energy expenditure related to activity quantified by duration and frequency. ${ }^{27}$ If total volume of PA is the main contributor in the relationship between mortality and PA, it stands to reason that our global score, which includes frequency, intensity and duration of PA to deduce PA levels, is appropriate for use in the estimation of the magnitude of a given effect.

A weakness of our study is the reliance on self-report for all covariates examined. This could introduce misclassification error, thereby affecting our estimates. However, in the NOWAC Study information on the use of $\mathrm{HT}^{20}$ and dietary habits, including alcohol habits, ${ }^{21,22}$ have been validated with good results. Furthermore, information on parity and duration of education has been compared to national registers with no statistically significant differences found. ${ }^{15}$ Self-reported information on height and weight has not yet been validated in the NOWAC Study, but ongoing research (unpublished) indicates a limited extent of misclassification bias. Despite certain limitations, self-reporting methods are considered adequate to deal with large sample sizes, practical to administer, and less expensive.
Disease or preliminary stages of a disease may inhibit a person's ability to be physically active, meaning that part of the observed association between PA level and mortality may be due to confounding or reverse causation. Restricting the analysis to women without a history of disease would have entailed exclusion of almost 12,000 women, and therefore we retained and adjusted for this information, in addition to performing a sensitivity analysis excluding them. The effect of PA on mortality remained consistent both in the sensitivity analysis and the stratified analysis according to CVD disease, diabetes mellitus and prevalent cancer. Thus, it is unlikely that our results might be due to low PA levels explaining prevalent disease, but more probably that prevalent diseases are explaining low PA levels. Residual confounding can never be excluded in non-randomized studies, however, other prospective studies support our findings.

Despite the limitations of this study, and the existence of a possible spurious component in the relationship between PA levels and mortality, the benefits of being physically active are biologically plausible. An increase from low PA levels up to a moderate PA level would entail an $11.5 \%$ reduction in all-cause mortality in women in Norway. PAF estimates depend on RRs and the proportion of exposure in a given population, which has to be considered when generalizing to other populations where low PA levels could differ. Our study design cannot determine a causal association, but our results indicate a significant trend of reduced mortality risk with increasing PA levels, which suggests such an association. The results of the present study suggest that PA level is an important lifestyle behaviour to target in public health strategies.

\section{Acknowledgments}

The authors thank the NOWAC Study staff and participants for their invaluable contributions to this study.

\section{Author contributions}

$\mathrm{KBB}$ carried out the statistical analysis and drafted the manuscript. TB contributed to the statistical analysis and interpretation of the data. EL is the principal investigator and designed the NOWAC Study. EW contributed with planning of the data analysis, interpretation of the data and critical revision of the manuscript. All authors read and approved the final manuscript.

\section{Disclosure}

The authors report no conflicts of interest in this work. 


\section{References}

1. Besson H, Ekelund U, Brage S, et al. Relationship between subdomains of total physical activity and mortality. Med Sci Sports Exerc. 2008; 40(11):1909-1915.

2. Oguma Y, Sesso HD, Paffenbarger RS Jr, Lee IM. Physical activity and all cause mortality in women: a review of the evidence. Br J Sports Med. 2002;36(3):162-172.

3. Crespo CJ, Palmieri MR, Perdomo RP, et al. The relationship of physical activity and body weight with all-cause mortality: results from The Puerto Rico Heart Health Program. Ann Epidemiol. 2002;12(8): 543-552.

4. Hu FB, Willet WC, Li T, Stampfer MJ, Colditz GA, Manson JE. Adiposity as compared with physical activity in predicting mortality among women. N Engl J Med. 2004;351(26):2694-2703.

5. Katzmarzyk PT, Church TS, Craig CL, Bouchard C. Sitting time and mortality from all causes, Cardiovascular Disease, and Cancer. Med Sci Sports Exerc. 2009;41(5):998-1005.

6. Leitzmann MF, Park Y, Blair A, et al. Physical activity recommendations and decreased risk of mortality. Arch Intern Med. 2007;167(22): 2453-2460.

7. Nocon M, Hiemann T, Muller-Riemenschneider F, Thalau F, Roll S, Willich SN. Association of physical activity with all-cause and cardiovascular mortality: a systematic review and meta-analysis. Eur J Cardiovasc Prev Rehabil. 2008;15(3):239-246.

8. Katzmarzyk PT, Mason C. The physical activity transition. J Phys Act Health. 2009;6(3):269-280.

9. Warburton D, Charlesworth S, Ivey A, Nettlefold L, Bredin S. A systematic review of the evidence for Canada's Physical Activity Guidelines for Adults. Int J Behav Nutr Phys Act. 2010;7:39.

10. Helsedirektoratet. Kostråd for å fremme folkehelsen og forebygge kroniske sykdommer. Metodologi og vitenskapelig kunnskapsgrunnlag. Nasjonalt råd for ernæring 2011. Oslo: Helsedirektoratet; 2011 [updated 01.03.2011; cited 2011]; 356]. Available from: http://www. helsedirektoratet.no/vp/multimedia/archive/00322/Kostr_d_for_ fremm_322509a.pdf.

11. WHO. 2008-2013 Action plan for the global strategy for the prevention and control of noncommunicable diseases. Geneva, Switzerland: WHO; 2008.

12. Lee IM, Skerrett PJ. Physical activity and all-cause mortality: what is the dose-response relation? Med Sci Sports Exerc. 2001;33 (Suppl 6):S459-S471.

13. Trolle-Lagerros Y, Mucci LA, Kumle M, et al. Physical activity as a determinant of mortality in women. Epidemiology. 2005;16(6):780-785.

14. Lund E, Dumeaux V, Braaten T, et al. Cohort Profile: The Norwegian Women and Cancer Study - NOWAC - Kvinner og kreft. Int J Epidemiol. 2008;37(1):36-41.

15. Lund E, Kumle M, Braaten T, et al. External validity in a populationbased national prospective study - the Norwegian Women and Cancer Study (NOWAC). Cancer Causes Control. 2003;14:1001(10)-1008.

16. Hjartåker A, Lund E, Bjerve KS. Serum phospholipid fatty acid composition and habitual intake of marine foods registered by a semiquantitative food frequency questionnaire. Eur J Clin Nutr. 1997; 51(11):736-742.

17. Hjartåker A, Lund E. Relationship between dietary habits, age, lifestyle, and socio-economic status among adult Norwegian women. The Norwegian Women and Cancer Study. Eur J Clin Nutr. 1998;52(8): 565-572.

Clinical Epidemiology

\section{Publish your work in this journal}

Clinical Epidemiology is an international, peer-reviewed, open access journal focusing on disease and drug epidemiology, identification of risk factors and screening procedures to develop optimal preventative initiatives and programs. Specific topics include: diagnosis, prognosis, treatment, screening, prevention, risk factor modification, systematic
18. Skeie G, Hjartåker A, Lund E. Diet among breast cancer survivors and healthy women. The Norwegian Women and Cancer Study. Eur J Clin Nutr. 2006;60(9):1046-1054.

19. Rockhill B, Newman B, Weinberg C. Use and misuse of population attributable fractions. Am J Public Health. 1998;88(1):15-19.

20. Waaseth M, Bakken K, Lund E. Patterns of hormone therapy use in the Norwegian Women and Cancer study (NOWAC) 1996-2005. Maturitas. 2009;63(3):220-226.

21. Hjartåker A, Andersen LF, Lund E. Comparison of diet measures from a food-frequency questionnaire with measures from repeated 24-hour dietary recalls. The Norwegian Women and Cancer Study. Public Health Nutr. 2007;10(10):1094-1103.

22. Parr C, Veierфd M, Laake P, Lund E, Hjartåker A. Test-retest reproducibility of a food frequency questionnaire (FFQ) and estimated effects on disease risk in the Norwegian Women and Cancer Study (NOWAC). Nutr J. 2006;5:4.

23. Woodcock J, Franco OH, Orsini N, Roberts I. Non-vigorous physical activity and all-cause mortality: systematic review and meta-analysis of cohort studies. Int J Epidemiol. 2011;40(1):121-138.

24. Baer HJ, Glynn RJ, Hu FB, et al. Risk factors for mortality in the nurses' health study: a competing risks analysis. Am J Epidemiol. 2011;173(3): 319-329.

25. Mathers C, Stevens G, Mascarenhas M. Global Health Risks: mortality burden of disease attributable to selected major risk. Switzerland: WHO; 2009. ISBN: 9789241563871. Available from: http://www.who.int/ healthinfo/global_burden_disease/GlobalHealthRisks_report_full.pdf.

26. Dam RMV, Li T, Spiegelman D, Franco OH, Hu FB. Combined impact of lifestyle factors on mortality: prospective cohort study in US women. BMJ. 2008;337:a1440. doi:10.1136/bmj.a1440.

27. Lee I-M, Blair SN, Manson JAE, Paffenbarger RS Jr, editors. Epidemiologic Methods in Physical Activity Studies. New York: Oxford University Press; 2009.

28. Autenrieth CS, Baumert J, Baumeister SE, et al. Association between domains of physical activity and all-cause, cardiovascular and cancer mortality. Eur J Clin Nutr. 2011;26(2):91-99.

29. Thune I, Furberg AS. Physical activity and cancer risk: dose-response and cancer, all sites and site-specific. Med Sci Sports Exerc. 2001; 33(Suppl 6):S530-S550.

30. Matthews CE, Jurj AL, Shu X-O, et al. Influence of exercise, walking, cycling, and overall nonexercise physical activity on mortality in Chinese women. Am J Epidemiol. 2007;165(12):1343-1350.

31. DiPietro L. Physical activity in aging. J Gerontolol A: Biol Sci Med Sci. 2001;56:13-22.

32. Sallis JF, Saelens BE. Assessment of physical activity by self-report: Status, limitations, and future directions. Res Q Exerci Sport. 2000; 71(Suppl 2):S1-S14.

33. Lagerros YT, Mucci LA, Bellocco R, Nyren O, Bälter O, Bälter KA. Validity and reliability of self-reported total energy expenditure using a novel instrument. Eur J Epidemiol. 2006;21(3):227-236.

34. Lamonte MJ, Ainsworth BE. Quantifying energy expenditure and physical activity in the context of dose response. Med Sci Sports Exerc. 2001;33(Suppl 6):S370-S378. 